\title{
Spanish adaptation and validation of the Child Food Security Survey Module (CFSSM-S)
}

\author{
Nithya Shankar-Krishnan ${ }^{1, *}$, Eva Penelo ${ }^{2,3}$, Albert Fornieles $\operatorname{Deu}^{2,4}$ and \\ David Sánchez-Carracedo' \\ 'Departament de Psicologia Clínica i de la Salut, Universitat Autònoma de Barcelona, Bellaterra (Cerdanyola del \\ Vallès), 08193, Barcelona, Spain: ${ }^{2}$ Departament de Psicobiologia i de Metodologia de les Ciències de la Salut, \\ Universitat Autònoma de Barcelona, Barcelona, Spain: ${ }^{3}$ Laboratori d'Estadística Aplicada, Universitat Autònoma de \\ Barcelona, Barcelona, Spain: ${ }^{4}$ Professor Serra Húnter, Universitat Autònoma de Barcelona, Barcelona, Spain
}

Submitted 1 March 2018: Final revision received 2 May 2018: Accepted 24 May 2018: First published online 12 July 2018

\begin{abstract}
Objective: The present study aimed to assess the: (i) internal structure of the Spanish Child Food Security Survey Module (CFSSM-S) with exploratory and confirmatory factor analysis (EFA and CFA); (ii) measurement invariance by gender, grade, weight status, socio-economic status (SES) and family affluence; and (iii) relationships with these external variables.

Design: A cross-sectional study was conducted. The CFSSM-S and other tools were employed to assess food insecurity, weight status, SES and family affluence, respectively.

Setting: A secondary school (grades 7-10) in the city of Terrassa in Catalonia, Spain. Subjects: Participants included adolescent boys and girls ( $n$ 426) aged $12-17$ years. Results: The cross-validation design with EFA and CFA captured a single factor, 'food insecurity'. The goodness-of-fit for the one-factor model with CFA (rootmean-square error of approximation $=0.038$, comparative fit index $=0.984$, Tucker-Lewis index $=0.979)$ and internal consistency $(\omega=0.95)$ were excellent. The measurement invariance indicated that CFSSM-S could be used across genders, grades, weight status, SES and family affluence. Only mean differences for SES and family affluence were found which showed a linear trend, indicating higher CFSSM-S scores for participants with lower SES and family affluence. Of participants, $1.9 \%$ experienced very low food security, $16.4 \%$ low food security and $81.7 \%$ were food secure.

Conclusions: The CFSSM-S is the first validated instrument to assess food insecurity with psychometric guarantees in Spanish adolescents. Researchers and health practitioners in Spain could use this self-reported questionnaire to gain more information about adolescent health in relation to food insecurity.
\end{abstract}

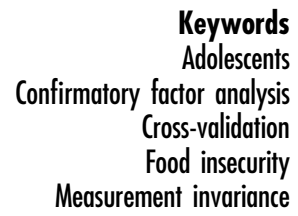

Keywords factor analysis Cross-validation Food insecurity Measurement invariance
The economic downturn in 2008 has resulted in food insecurity becoming a huge public health burden in Europe $^{(1,2)}$. The aftermath of the crisis led to an increase in unemployment and poverty in developed countries like Spain as well as contributed to issues in food affordability and nutritional status ${ }^{(3)}$. In Spain, the gap between upper and lower social classes has widened significantly and child poverty rates are considerably higher than the rich world average ${ }^{(4)}$. The onus falls on notable reductions in social benefits and meal subsidies, which have greatly affected the well-being of individuals in Spain, particularly children $^{(5)}$. Specifically, $20 \%$ of the population in Catalonia, Spain are living under the poverty line ${ }^{(6)}$. This region also reported an increase of $23 \%$ in child poverty in 2017 , with
261970 children living in severe poverty ${ }^{(7)}$. There have been studies that have explored the relationship between poverty and food insecurity and it has been noted that higher levels of food insecurity are experienced in households living near or under the poverty line ${ }^{(8)}$. However, it is not necessary that all households living in poverty are food insecure or all food-insecure households face poverty ${ }^{(9)}$. It is thereby essential to understand the definition of food insecurity.

Food insecurity does not simply refer to the lack of access to food. It is defined as the limited or uncertain availability of nutritionally adequate and safe foods or limited or uncertain ability to acquire acceptable foods in socially acceptable ways, ${ }^{,(0,11)}$. Considering high food 
prices and low income, food-insecure individuals often find it difficult to afford nutritionally essential foods prepared under sanitary conditions ${ }^{(12,13)}$. Moreover, food insecurity is paradoxical, as both forms of malnutrition namely hunger and overnutrition - can exist within foodinsecure households ${ }^{(14)}$.

Food insecurity can be measured indirectly through household income surveys or dietary interviews. However, one of the most reliable methods of direct assessment is through food insecurity questionnaires. The earliest instruments that measure household food insecurity include the Radimer-Cornell, which focuses on food quality and quantity in households ${ }^{(15)}$. Since then other instruments have been developed, such as the Household Food Security Access Scale (HFIAS) ${ }^{(16)}$ by the US Agency for International Development and the Household Food Security Survey Module (US-FSSM) ${ }^{(17)}$ by the US Department of Agriculture.

There are household food insecurity questionnaires adapted and validated in different languages, including Spanish. The Spanish version of the US-FSSM ${ }^{(18)}$ particularly addresses Hispanic American households, while the Latin American and Caribbean Scale (ELCSA) ${ }^{(19)}$ has been developed to focus on food insecurity issues in Latin America and the Caribbean. Neither of these instruments has been validated in Spain.

In addition, all these instruments are focused on adults and their understanding of the food insecurity situation in the households, but do not represent the individual experiences of children, especially adolescents ${ }^{(20)}$. The Child Food Security Survey Module $(\mathrm{CFSSM})^{(21)}$, part of the US Department of Agriculture survey instruments, is a valid and reliable tool that can effectively measure adolescents' perceptions of food insecurity in their households. The instrument is unique in its ability to be self-reported by adolescents and does not require the presence of adults. The specific aim of the CFSSM was to determine children's perceptions from the eighteen-item US-FSSM instrument, suitably modify the items to make the language and formatting easier for older children and retain the originality of the items ${ }^{(21)}$.

In this regard, based on qualitative interviews with children, nine items were chosen from the US-FSSM. There were two major changes made in the CFSSM. The first change was introduced in the response sets of the instrument, which maintained a multiple-choice format, but the responses were set as 'all/most of the time', 'some of the time' and 'none of the time', which makes it easier for children to answer. The second change was in the time frame of the items. The US-FSSM suggests a 12-month reference period. However, the authors shortened the time frame for the CFSSM to 1 month, considering possible recall bias in children ${ }^{(21)}$. The nine-item CFSSM was piloted in a multiracial sample of 467 adolescent children aged $12-15$ years in a secondary school in Mississippi, USA. The Rasch model was employed to analyse the responses to the food insecurity items. The reliability reported for the
CFSSM was considered modest but acceptable by the authors. Both validity and reliability were determined to be adequate for both genders and for older children $(\geq 12$ years) but unsuitable for younger children ${ }^{(21)}$.

Considering the lack of previous validated self-reported food insecurity instruments for adolescents in Spain, the main objectives of our study were to determine the: (i) internal structure of the Child Food Security Survey Module-Spanish version (CFSSM-S) with exploratory and confirmatory factor analysis (EFA and CFA) in a sample of Spanish adolescents; (ii) measurement invariance across gender, grade, weight status, socio-economic status (SES) and family affluence; and (iii) relationship of food insecurity scores with external variables (gender, grade, weight status, SES and family affluence). For the latter objective, no differences are expected by gender as not many studies have determined gender differences in food insecurity. Further, the existing few studies that have found differences have been in a developing country ${ }^{(22)}$ and a rural community ${ }^{(23)}$. With respect to age or grade, no differences are expected. Adolescents are considered at a higher risk of food insecurity than younger children ${ }^{(24)}$, but our study includes only adolescents. There have been contradictory results regarding weight status and food insecurity, since some studies have determined an association between overweight and food insecurity in adolescents $^{(25,26)}$, while others have found no associations ${ }^{(27,28)}$. Finally, food insecurity scores are anticipated to be higher in participants with lower SES and family affluence, as the risk of food insecurity increases in economically disadvantaged classes ${ }^{(29)}$.

\section{Methods}

\section{Participants}

The initial sample consisted of 475 adolescents from a secondary public school in Terrassa, the third most populous (>200000 inhabitants) urban industrial city in Catalonia $^{(30)}$ (Spain). During the assessment, due to absenteeism ( $n$ 46) and lack of consent (parental or self; $n$ 3), 426 adolescents (53\% boys and $47 \%$ girls) took part in the study. The age of the participants ranged from 12 to 17 years (mean 13.8 (SD 1.2) years). Adolescents from the four years of Compulsory Secondary Education as per the Spanish education system (equivalent to US grades 7-10) took part in the present study. Participants were roughly distributed across grades: 26.1, 28.6, 25.6 and $19.7 \%$ in grade $7,8,9$ and 10 , respectively. The origin of participants included $85.2 \%$ Spanish, $4.2 \%$ mixed origins, $3.8 \%$ Hispanic Americans, 2.8\% Europeans, $2 \cdot 1 \%$ other origins, $1.2 \%$ Sub-Saharan Africans and $0.7 \%$ North Africans. The following categories of $\mathrm{SES}^{(31)}$ were derived in participants: high, $14.0 \%$; medium-high, $24.5 \%$; medium, $24.7 \%$; medium-low, 27.1\%; low, $9.7 \%$ (as explained in the 'Measures' section, 'Socio-economic status' subsection). 


\section{Measures}

Child Food Security Survey Module-Spanish version The CFSSM-S contains nine items focusing on participants' perceptions of food insecurity in the households and their worries such as running out of food, eating only cheap foods, not being able to eat a balanced diet, eating less, cutting down on portions of food, skipping meals, going hungry and not eating for a whole day. The instructions, format, response sets and scoring of the CFSSM-S (see online supplementary material) were identical to the original instrument. In this regard, the responses were based on a Likert scale. Affirmative responses of 'mucho' (a lot) and ' $a$ veces' (sometimes) were scored 1 , while negative response 'nunca' (never) was scored 0 . The scores were added and higher scores indicated food insecurity. The food security classification as per the original study ${ }^{(21)}$ and the US Department of Agriculture's new nomenclature ${ }^{(32)}$ established the following categories: $0-1=$ food secure; $2-5=$ low food security; and 6-9=very low food security. The reference period was set to 12 months as our sample included only adolescents.

\section{Socio-economic status}

We used the Four-Factor Index of Social Status ${ }^{(31)}$ to determine SES of the household using a weighted average of each parent's education and occupation level. It takes account of education scores ranging from 1 to 7 (i.e. primary school to graduate degree) and occupation scores ranging from 1 to 9 (i.e. unemployed to higher executives). According to Hollingshead, SES was calculated by adding Education score $\times 3$ and Occupation score $\times 5$; then, total scores, which ranged from 8 to 66 , were categorized into five different levels: high (55-66), medium-high (40-54), medium (30-39), medium-low (20-29) and low (8-19).

\section{Family Affluence Scale III}

The Spanish version of the Family Affluence Scale III

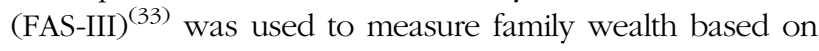
material assets. Although parental income based on education and occupation is a good indicator of social class, the FAS is considered an effective predictor of household affluence, since adolescents often face difficulties in reporting the measure of family wealth ${ }^{(34)}$. It consists of six questions asking participants about the possession of a car and dishwasher, number of bedrooms, bathrooms, computers/ mobile phones and vacations per year. A four-point ordinal scale ranging from 0 to 3 was used for the six questions. The scores were added. The range of responses varies from 0 to 13 and the cut-off points set for purchasing power were 0-6 for low, 7-9 for medium and 10-13 for high.

\section{Weight status}

Height in centimetres was measured by a SECA portable stadiometer, model $214(20-207 \mathrm{~cm}$; accuracy range of $0.1 \mathrm{~cm}$ ) and weight in kilograms using SECA portable scales, model 8777021094 (0-200 kg; accuracy range of $0.1 \mathrm{~kg}$ ). Height and weight of participants were taken in order to achieve the same consecutive measurements. Participants were requested to remove heavy articles of clothing including shoes, jackets, watches and mobile phones. Considering the weight of clothing worn by participants, corrected weight was assessed by subtracting $0.9 \mathrm{~kg}$ from males and $0.7 \mathrm{~kg}$ from females. BMI $Z$-scores and weight status using the WHO 2007 growth reference criteria $^{(35)}$ were calculated based on participants' height, weight and age.

\section{Procedure}

The Community and Health Services of the City Council of Terrassa facilitated the access to the secondary school. To ensure maximum participation, all details concerning the study were previously coordinated with the executive team of the secondary school. The administration of questionnaires and anthropometric measurements were completed during normal class hours within four days ( $1 \mathrm{~h}$ per class) in April 2017. Participants completed a paper-andpencil booklet with a battery of validated questionnaires and socio-economic data. The research staff included postgraduate students who were trained to administer questionnaires, take the anthropometric measurements and provide instructions to participants. Following a standardized procedure, anthropometric measurements were carried out in situ in a private room near the classroom where the questionnaires were administered.

The adaptation of the CFSSM-S from English to Spanish was conducted in three main steps following the International Test Commission Guidelines ${ }^{(36,37)}$. First, an expert, native in the target language (Spanish) and possessing a good level of the source language (English), translated the CFSSM instrument from English to Spanish. Second, the translated document was examined by a panel of experts comprising various academics from the field of eating behaviours and disordered eating. The expert panel compared the translated version with the original one, taking account of parameters such as item format, grammar and writing, culture, etc. by using a scoring based on a Likert scale of $1-5$. In the case of discrepancies, the items were remodified by the panel that further introduced minor changes to the translated document. Further, the translated document was administered to a group of fifteen adolescent boys and girls aged 12-16 years to make sure that it was easily comprehensible. Minor changes were made to the document. Finally, the official translation services of our university further corrected and approved the translated document according to the standards of the Council of Europe's Common European Framework of Reference for Languages.

\section{Statistical analysis}

Analyses were conducted with the statistical software packages Mplus version 8 and IBM SPSS Statistics 
version 23. First, to determine the internal structure of the nine CFSSM-S items a cross-validation design was employed, by splitting the sample randomly in two subsamples. In the first sub-sample, EFA with extraction of one and two factors was conducted, with geomin rotation for the two-factor solution. And in the second sub-sample, CFA was conducted to test if the EFA solutions could be replicated. For both analyses, the WSLMV (weighted least-squares means and variance) method of estimation for categorical items was applied, using the polychoric correlation matrix, given that responses to items were dichotomous. Goodness-of-fit was evaluated with the common indices ${ }^{(38)}: \chi^{2}$, root-mean-square error of approximation (RMSEA), comparative fit index (CFI) and TuckerLewis index (TLI). We followed the usual cut-off points ${ }^{(39)}$. RMSEA $<0.08$ and CFI and TLI $>0.90$ would indicate acceptable fit, and RMSEA $<0.06$ and CFI and TLI $>0.95$ would indicate excellent fit.

Once dimensionality was established in the whole sample, assessment of measurement invariance was conducted to determine whether the questionnaire measured the same trait across different subgroups. Measurement invariance was conducted across the following groups of responses: gender, grade, weight status, SES and FAS. For variables with low $(n<100)$ or very unequal sample sizes in some categories, the following groupings were considered: grade 7 and $8 v$. grade 9 and 10; overweight and obesity $v$. normal weight (data for participants with underweight were excluded because of very low sample size, $n$ 8); high, medium-high and medium $v$. medium-low and low SES; and low and medium $v$. high FAS. Measurement invariance involved four sequential steps: (i) configural (equal form); (ii) equivalence of factor loadings (metric or weak measurement invariance); (iii) item thresholds (scalar or strong measurement invariance); and (iv) item uniquenesses (strict measurement invariance). Model identification for each step was established using the fixed-factor method $^{(40)}$. Comparison between nested models was conducted with the scaled difference $\chi^{2}$ test (DIFFTEST option of Mplus; $\alpha$ level set at 0.05).

Next, internal consistency was assessed with the omega coefficient $^{(41)}$. Finally, once measurement invariance was established, comparisons of observed CFSSM-S scores among gender, grade, weight status, SES and FAS was conducted with one-way ANOVA.

\section{Results}

The mean BMI of the total sample was 21.11 (SD 3.95) $\mathrm{kg} / \mathrm{m}^{2}$. As per the WHO growth reference criteria for BMI-for-age ${ }^{(35)}$, participants in the present study were classified as: severely thin, $0.5 \%$; thin, $1.5 \%$; normal, $67.7 \%$; overweight, $21.6 \%$; and obese, $8.7 \%$.

\section{Internal structure: dimensionality. Internal consistency}

Descriptive statistics for the nine CFSSM-S items are displayed in Table 1. Mean values ranged from 0.01 (SD $0 \cdot 10$ ) to $0 \cdot 26$ (SD 0.44). There were no missing values for any item.

Regarding the cross-validation strategy, no differences were observed for gender, grade, weight status, SES and FAS between the two random sub-samples $(P \geq 0 \cdot 410)$. Two eigenvalues for EFA in the first sub-sample ( $n$ 205) were above 1 (5.89 and 1.24), with the scree test suggesting the extraction of only one factor. The one-factor model with EFA showed acceptable fit $\left(\chi_{(27)}^{2}=66 \cdot 0\right.$, RMSEA $=0.084, \mathrm{CFI}=0.952$, TLI $=0.937)$; all factor loadings were above $0.30(0.54,0.97)$ and statistically significant $(P<0.05)$. The one-factor model with CFA in the second sub-sample ( $n$ 221) showed excellent fit $\left(\chi_{(27)}^{2}=29 \cdot 5, \quad\right.$ RMSEA $=0.021, \quad$ CFI $=0.995, \quad$ TLI $\left.=0.993\right)$, with all standardized factor loadings above $0.30(0 \cdot 71$, $0 \cdot 96)$ and statistically significant $(P<0 \cdot 05)$. Moreover, we also examined a two-factor solution in both sub-samples. The two-factor model with EFA and geomin rotation in the first sub-sample showed better fit than the onefactor model $\left(\chi_{(19)}^{2}=22 \cdot 2, \quad\right.$ RMSEA $=0.029, \quad$ CFI $=0.96$, $\mathrm{TLI}=0.993)$, but some parameters were unsatisfactory and a simple structure was not attained: item 7 showed a standardized factor loading above 1, items 2 and 5 showed cross-loadings (factor loadings above 0.30 in both factors) and the factor correlation was high

Table 1 Descriptive statistics of items and standardized parameters for the one-factor solution obtained by confirmatory factor analysis (model F in Table 2): Spanish adaptation and validation of the Child Food Security Survey Module (CFSSM-S)

\begin{tabular}{|c|c|c|c|c|c|}
\hline Item & Mean & SD & Factor loading & Item threshold & Uniqueness \\
\hline 1. Worry & 0.26 & 0.44 & 0.73 & 0.63 & 0.46 \\
\hline 2. Food run out & 0.08 & 0.26 & 0.79 & 1.44 & 0.37 \\
\hline 3. Cheap food & 0.15 & 0.36 & 0.78 & 1.04 & 0.39 \\
\hline 4. Balanced meal & 0.08 & 0.27 & 0.80 & 1.42 & 0.36 \\
\hline 5. Eat less & 0.05 & 0.22 & 0.95 & 1.65 & 0.10 \\
\hline 6. Meals cut & 0.06 & 0.24 & 0.84 & 1.57 & 0.29 \\
\hline 7. Skip a meal & 0.03 & 0.16 & 0.93 & 1.95 & 0.16 \\
\hline 8. Hungry & 0.07 & 0.26 & 0.75 & 1.46 & 0.44 \\
\hline 9. Not eat for a whole day & 0.01 & $0 \cdot 10$ & 0.97 & $2 \cdot 35$ & 0.05 \\
\hline Total score & 0.78 & 1.45 & & & \\
\hline Internal consistency ( $\omega$ coefficient) & & & 0.95 & & \\
\hline
\end{tabular}


$(r=0 \cdot 62)$. However, we tried to replicate this two-factor model with CFA in the second sub-sample, by allocating each item on to the factor with a higher factor loading (items 1-3-4 and 2-5-6-7-8-9). This two-factor model with CFA and correlated factors in the second subsample did not exhibit improved fit when compared with the one-factor solution $\left(\chi_{(18)}^{2}=28 \cdot 1, \quad\right.$ RMSEA $=0 \cdot 019$, $\mathrm{CFI}=0.996, \quad \mathrm{TLI}=0.994)$ and factor correlation was excessively high $(r=0.92)$, indicating overlap between factors $^{(39)}$

Considering these results, we selected the one-factor model. Table 1 shows the parameters for this model with CFA in the whole sample ( $n$ 426). Factor loadings (all statistically significant, $P<0.05$ ) and item thresholds ranged, respectively, from 0.73 and 0.63 (item 1) to 0.97 and 2.35 (item 9). Goodness-of-fit (model $\mathrm{F}$ in Table 2, which is commented upon in the 'Internal structure: measurement invariance' subsection) was excellent $\left(\chi_{(27)}^{2}=43 \cdot 4\right.$, RMSEA $\left.=0.038, \mathrm{CFI}=0.984, \mathrm{TLI}=0.979\right)$.

Omega internal consistency value derived from CFA in the whole sample was excellent $(\omega=0.95)$.

\section{Internal structure: measurement invariance}

Table 2 displays the results of CFA baseline models by group and measurement invariance analyses. Fit for baseline models (model 0) and for configural invariance across groups of responses (model 1) was satisfactory (RMSEA $\leq 0.055, \quad$ CFI $\geq 0.942, \quad$ TLI $\geq 0.923$ ). Full weak (equivalence of factor loadings), strong (equivalence of item thresholds) and strict (equivalence of uniqueness invariance) was achieved $(P \geq 0 \cdot 105)$ for all the groups of responses considered. These findings provide support for the equivalence of CFSSM-S scores across gender, grade, weight status, SES and FAS, and comparisons among these groups will be readily interpretable.

\section{Relationship with external variables}

Direct CFSSM-S scores were calculated by applying the simple weighting method $^{(42)}$ given the adequate results for CFA and measurement invariance analyses. Table 3 shows descriptive statistics and comparison among groups. No differences were found by gender $(P=0 \cdot 344)$, grade $(P=0.765)$ and weight status $(P=0.061)$, whereas

Table 2 Fit indices for measurement invariance analyses across gender, grade, weight status, socio-economic status (SES) and family affluence (FAS): Spanish adaptation and validation of the Child Food Security Survey Module (CFSSM-S)

\begin{tabular}{|c|c|c|c|c|c|c|c|c|c|c|c|}
\hline \multirow[b]{2}{*}{ Group } & \multirow[b]{2}{*}{ Model fit and invariance } & \multicolumn{6}{|c|}{ Goodness-of-fit index } & \multicolumn{4}{|c|}{ Comparison } \\
\hline & & $x^{2}$ & df & $\mathrm{CFI}$ & TLI & RMSEA & $90 \% \mathrm{Cl}$ & Models & $\Delta x^{2 *}$ & $\Delta \mathrm{df}$ & $P$ \\
\hline \multirow[t]{6}{*}{ Gender } & A0a: males ( $n$ 226) & 33.5 & 27 & 0.988 & 0.984 & 0.033 & $0.000,0.064$ & & & & \\
\hline & A0b: females $(n 200)$ & 33.9 & 27 & 0.991 & 0.987 & 0.036 & $0.000,0.069$ & & & & \\
\hline & A1: configural (equal form) & 67.4 & 54 & 0.989 & 0.986 & 0.034 & $0.000,0.058$ & & & & \\
\hline & A2: A1 plus equal factor loadings (weak invariance) & $70 \cdot 0$ & 62 & 0.993 & 0.992 & 0.025 & $0.000,0.050$ & $\mathrm{~A} 2$ v. A1 & 7.5 & 8 & 0.483 \\
\hline & A3: A2 plus equal thresholds (strong invariance) & $75 \cdot 8$ & 70 & 0.995 & 0.995 & 0.020 & $0.000,0.045$ & A3 v. A2 & 3.8 & 8 & 0.878 \\
\hline & A4: A3 plus uniquenesses free (strict invariance) $\dagger$ & $73 \cdot 3$ & 61 & 0.990 & 0.988 & 0.031 & $0.000,0.054$ & A3 v. A4† & 6.1 & 9 & 0.728 \\
\hline \multirow[t]{6}{*}{ Grade } & B0a: 01-02 (n 233) & $26 \cdot 4$ & 27 & 1.000 & 1.002 & 0.000 & $0.000,0.050$ & & & & \\
\hline & B0b: 03-04 (n 193) & $42 \cdot 8$ & 27 & 0.970 & 0.960 & 0.055 & $0.019,0.085$ & & & & \\
\hline & B1: configural (equal form) & 67.3 & 54 & 0.987 & 0.983 & 0.034 & $0.000,0.058$ & & & & \\
\hline & B2: B1 plus equal factor loadings (weak invariance) & $69 \cdot 8$ & 62 & 0.993 & 0.991 & 0.024 & $0.000,0.049$ & $\mathrm{~B} 2$ v. B1 & 7.8 & 8 & 0.449 \\
\hline & B3: B2 plus equal thresholds (strong invariance) & $79 \cdot 8$ & 70 & 0.991 & 0.990 & 0.026 & $0.000,0.049$ & B3 v. B2 & $11 \cdot 2$ & 8 & $0 \cdot 190$ \\
\hline & B4: B3 plus uniquenesses free (strict invariance) $\dagger$ & $79 \cdot 3$ & 61 & 0.983 & 0.980 & 0.037 & $0.000,0.059$ & B3 v. B4† & $6 \cdot 3$ & 9 & 0.705 \\
\hline \multirow[t]{6}{*}{ Weight status } & C0a: normal weight ( $n$ 279) & 44.6 & 27 & 0.942 & 0.923 & 0.048 & $0.020,0.073$ & & & & \\
\hline & COb: overweight and obesity ( $n$ 125) & $26 \cdot 9$ & 27 & 1.000 & 1.000 & 0.000 & $0.000,0.069$ & & & & \\
\hline & C1: configural (equal form) & 71.7 & 54 & 0.978 & 0.971 & 0.040 & $0.000,0.063$ & & & & \\
\hline & C2: C1 plus equal factor loadings (weak invariance) & $78 \cdot 1$ & 62 & 0.980 & 0.977 & 0.036 & $0.000,0.058$ & $\mathrm{C} 2$ v. C1 & $10 \cdot 4$ & 8 & 0.235 \\
\hline & C3: C2 plus equal thresholds (strong invariance) & $87 \cdot 2$ & 70 & 0.979 & 0.978 & 0.035 & $0.000,0.056$ & C3 v. C2 & $10 \cdot 0$ & 8 & 0.267 \\
\hline & C4: C3 plus uniquenesses free (strict invariance) $\dagger$ & $74 \cdot 0$ & 61 & 0.984 & 0.981 & 0.032 & $0.000,0.056$ & $\mathrm{C} 3$ v. C4† & 14.5 & 9 & 0.105 \\
\hline \multirow[t]{6}{*}{ SES } & D0c: high, medium-high and medium ( $n$ 266) & 28.4 & 27 & 0.998 & 0.998 & 0.014 & $0.000,0.051$ & & & & \\
\hline & D0e: medium-low and low ( $n$ 155) & $37 \cdot 0$ & 27 & 0.953 & 0.937 & 0.049 & $0.000,0.085$ & & & & \\
\hline & D1: configural (equal form) & $64 \cdot 1$ & 54 & 0.991 & 0.988 & 0.030 & $0.000,0.055$ & & & & \\
\hline & D2: D1 plus equal factor loadings (weak invariance) & $75 \cdot 0$ & 62 & 0.986 & 0.984 & 0.032 & $0.000,0.055$ & $\mathrm{C} 2$ v. $\mathrm{C} 1$ & 9.0 & 8 & 0.343 \\
\hline & D3: D2 plus equal thresholds (strong invariance) & $85 \cdot 0$ & 70 & 0.984 & 0.984 & 0.032 & $0.000,0.054$ & C3 v. C2 & 11.4 & 8 & 0.180 \\
\hline & D4: D3 plus uniquenesses free (strict invariance) $\dagger$ & $75 \cdot 0$ & 61 & 0.985 & 0.983 & 0.033 & $0.000,0.056$ & C3 v. C4† & $12 \cdot 4$ & 9 & 0.192 \\
\hline \multirow[t]{7}{*}{ FAS } & E0a: low and medium ( $n$ 246) & $35 \cdot 2$ & 27 & 0.981 & 0.975 & 0.035 & $0.000,0.064$ & & & & \\
\hline & EOb: high ( $n$ 180) & $29 \cdot 3$ & 27 & 0.995 & 0.994 & 0.022 & $0.000,0.063$ & & & & \\
\hline & E1: configural (equal form) & 64.6 & 54 & 0.989 & 0.985 & 0.030 & $0.000,0.055$ & & & & \\
\hline & E2: E1 plus equal factor loadings (weak invariance) & $66 \cdot 8$ & 62 & 0.995 & 0.994 & 0.019 & $0.000,0.046$ & $\mathrm{E} 2$ v. E1 & $6 \cdot 8$ & 8 & 0.555 \\
\hline & E3: E2 plus equal thresholds (strong invariance) & 74.5 & 70 & 0.995 & 0.995 & 0.017 & $0.000,0.044$ & E3 v. E2 & $7 \cdot 7$ & 8 & 0.461 \\
\hline & E4: E3 plus uniquenesses free (strict invariance) $\dagger$ & 67.5 & 61 & 0.993 & 0.992 & 0.022 & $0.000,0.048$ & E3 v. E4† & 9.1 & 9 & 0.431 \\
\hline & F: Final model (overall sample; $n$ 426) & 43.4 & 27 & 0.984 & 0.979 & 0.038 & $0.014,0.058$ & & & & \\
\hline
\end{tabular}

CFI, comparative fit index; TLI, Tucker-Lewis index; RMSEA, root-mean-square error of approximation.

${ }^{*} \Delta x^{2}$ based on DIFFTEST approach obtained from Mplus (scaled difference $x^{2}$ test for WLSMV (weighted least-squares means and variance) method of estimation).

†Test for invariance of uniquenesses for categorical indicators proceeds backwards (for more details, see Muthén and Muthén ${ }^{(51)}$; also, e.g., http://www. lesahoffman.com/CLP948/CLP948_Lecture07_Invariance.pdf). 
Table 3 Descriptive statistics for the total CFSSM-S score and comparison by gender, grade, weight status, socio-economic status (SES) and family affluence (FAS): Spanish adaptation and validation of the Child Food Security Survey Module (CFSSM-S)

\begin{tabular}{|c|c|c|c|c|c|c|c|c|c|}
\hline \multicolumn{2}{|c|}{ CFSSM-S total score (0-9) } & \multicolumn{3}{|c|}{ Descriptive statistics } & \multicolumn{3}{|c|}{ Comparison } & \multicolumn{2}{|c|}{ Polynomial contrasts } \\
\hline Variable & Level & Mean & $\mathrm{SD}$ & $n$ & $F$ & df & $P$ & $\mathrm{LT}(P)$ & QT $(P)$ \\
\hline \multirow[t]{2}{*}{ Gender } & Males & 0.72 & 1.44 & 226 & 0.90 & 1424 & 0.344 & & \\
\hline & Females & 0.85 & 1.46 & 200 & & & & & \\
\hline \multirow[t]{4}{*}{ Grade } & 01 & 0.78 & $1 \cdot 30$ & 111 & 0.38 & 3422 & 0.765 & & \\
\hline & 02 & 0.89 & 1.59 & 122 & & & & & \\
\hline & 03 & 0.69 & 1.33 & 109 & & & & & \\
\hline & 04 & 0.74 & 1.58 & 84 & & & & & \\
\hline \multirow[t]{3}{*}{ Weight-status } & Normal weight & 0.64 & $1 \cdot 12$ & 279 & 3.54 & $1401^{*}$ & 0.061 & & \\
\hline & Overweight & 1.07 & 1.96 & 89 & & & & & \\
\hline & Obesity & 0.92 & 1.44 & 36 & & & & & \\
\hline \multirow[t]{5}{*}{ SES } & High & 0.20 & 0.64 & 59 & 13.53 & $1416^{*}$ & $<0.005$ & $<0.005$ & 0.031 \\
\hline & Medium-high & 0.36 & 0.73 & 103 & & & & & \\
\hline & Medium & 0.81 & 1.71 & 104 & & & & & \\
\hline & Medium-low & 0.96 & $1 \cdot 25$ & 114 & & & & & \\
\hline & Low & 1.95 & $2 \cdot 22$ & 41 & & & & & \\
\hline \multirow{3}{*}{ FAS } & Low & 1.37 & 1.77 & 52 & $11 \cdot 15$ & $1423^{*}$ & 0.001 & $<0.005$ & 0.771 \\
\hline & Medium & 0.94 & 1.48 & 194 & & & & & \\
\hline & High & 0.43 & 1.22 & 180 & & & & & \\
\hline
\end{tabular}

$\mathrm{LT}$, linear trend; $\mathrm{QT}$, quadratic trend.

Significant $P$ values $(P<0.05)$ are indicated in bold font.

*Df corrected for non-homogeneity of variances (Levene's test).

CFSSM-S scores differed for SES and FAS $(P \leq 0 \cdot 001)$ showing a linear trend $(P \leq 0.05)$ : the lower the SES and FAS, the higher the CFSSM-S scores. Additionally, for SES, a slightly but statistically significant quadratic trend was also observed $(P=0.031)$, because the increase in CFSSM-S between participants with medium-low and low SES was higher than that for the remaining contiguous SES levels.

\section{Frequency of food insecurity}

Following the food security classification established in the original instrument ${ }^{(21)}$ and according to the US Department of Agriculture's new nomenclature ${ }^{(32)}, 1.9 \%$ of participants experienced very low food security, $16.4 \%$ had low food security and $81.7 \%$ were found to be food secure; no differences were found by gender $(P=0.540)$ or grade $(P=0.812)$. As in Table $1,26 \%$ of participants worried a lot or sometimes about food running out, $8 \%$ ran out of food, $15 \%$ consumed cheap foods, $8 \%$ could not eat a balanced meal, $5 \%$ had to eat less, $6 \%$ had to cut the size of their meals, $3 \%$ had to skip a meal, $7 \%$ were hungry and $1 \%$ did not eat for a whole day.

\section{Discussion}

The current study examined the validity of the CFSSM-S scores in terms of internal structure, measurement invariance and relationship with external variables. Our study is the first in Spain to carry out the adaptation and validation of a self-reported food insecurity questionnaire for adolescents. The results indicate that this instrument is valid and reliable for measuring perceptions of food insecurity adequately in Spanish adolescents.
The first objective was to examine the internal structure of the instrument. Our results showed that a one-factor model obtained a better fit, supporting the unidimensionality of the CFSSM-S and that all nine items belong to one factor, namely 'food insecurity'. We found that the endorsement of items in our sample was similar to the original instrument ${ }^{(21)}$; moreover, the ordering by groups of items based on threshold estimates in our CFA was almost identical to those obtained in the CFSSM with the Rasch model. Item 1, the least severe item of food insecurity, received the maximum number of affirmative responses. Worry or uncertainty about food was an expected psychological response from participants who were anxious about maintaining sufficient food supplies in their household ${ }^{(43)}$. In our study, the second and third most endorsed items, items 3 and 4 about 'consumption of cheap food' and 'lack of balanced diets', respectively, indicate that the adolescents were aware of the differences in diet quality. This affirms the fact that adolescents despite their knowledge about diet quality may have less control over the management of food resources at home ${ }^{(21)}$. As expected, item 9 was the most severe indicator of food insecurity that questions participants about not eating for a whole day, thus depicting insufficient food intake, and it was also the item which was least endorsed in both studies.

The second objective of the current study was to examine the measurement invariance of the instrument. The results indicate that this instrument can be used for both boys and girls, within a wide age range of adolescence (12-17 years) and for different levels of weight status, SES and FAS. This ensures that the instrument is measuring the same construct across these groups, providing support for making comparisons among these groups in a readily interpretable way. 
As equivalence for these groups had been established, further we proceeded with our third objective, which was to study the mean differences in food insecurity scores of the groups. The results indicate that there were no differences with gender. There are very few studies focused on gender differences in food insecurity. These studies indicate that both adolescent boys and girls were equally likely to experience severe food insecurity in their households, although girls were more likely to report food insecurity than boys ${ }^{(22,23)}$. Further, gender differences were found to be more prominent in severely foodinsecure households ${ }^{(22)}$. However, these studies were conducted in a rural community and a developing country while our sample included adolescents from a large city in the Barcelona area (Spain), which could explain the lack of gender differences. We concur that more studies are required to determine the association between gender and food insecurity. With regard to age, there have been studies indicating that food insecurity is more common in older children than younger children ${ }^{(24)}$. Food insecurity is notably higher in households with adolescents because parents often protect younger children ${ }^{(44)}$. Our study included only adolescents; hence, as expected, we were unable to find these differences based on age or grade.

Regarding weight status, our results are in alignment with previous studies ${ }^{(45,46)}$ indicating that even though there are no statistically significant differences compared with the normal group, the overweight and obese groups showed slightly higher food insecurity scores. Weight status has been a highly debated subject in the field of food insecurity. There have been inconsistent findings about the association of overweight and obesity with food insecurity ${ }^{(27)}$. However, some studies have confirmed this association in foodinsecure adolescents aged $12-17$ years ${ }^{(25,26)}$. Food-insecure households in general consume cheap, energy-dense foods and experience intermittent patterns of eating less when resources are limited and overeating when resources are readily available ${ }^{(47)}$. This results in drastic changes in food intake, which in turn could contribute to weight gain ${ }^{(48)}$. With respect to SES and FAS, our results showed that food insecurity scores increased with lower SES and FAS. These results are in alignment with previous studies that indicate food insecurity is associated with low SES ${ }^{(29,49)}$ and greater material hardships ${ }^{(50)}$.

Based on the food security classification of the original instrument ${ }^{(21)}$ and the US Department of Agriculture's new nomenclature ${ }^{(32)}, 1.9,16.4$ and $81.7 \%$ of participants in our study and $5.1,18.4$ and $76.7 \%$ in the original study belonged to the groups 'very low food security', 'low food security' and 'food secure', respectively. Our results indicate that $18.3 \%$ of participants experienced food insecurity, which is a fairly high percentage for a developed country like Spain. It would be important to emphasize that $26 \%$ of adolescents in our study worried about food, $15 \%$ considered the quality of their food to be cheap, $7 \%$ have been hungry and 3\% skipped meals. Although the results in both studies are very similar, there are slight differences in the 'very low food security' group. There are more participants with 'very low food security' in the original study compared with ours. Our sample consisted of a majority of participants from medium and medium-low SES, from the large metropolitan city of Terrassa, which could explain the reduced number of participants with 'very low food security'. Although the original study did not provide socio-economic information of their participants, the data were collected from a local school in the state of Mississippi, which reports the highest prevalence rate of food insecurity in the USA ${ }^{(9)}$. This could explain the differences in the 'very low food security' group in both studies. However, the results of the prevalence rate of food insecurity in our study should be considered with caution, given that our sample is not representative of the entire population. It is important to remember that the main objective of the study was to determine the internal structure of the CFSSM-S, measurement invariance across different variables and its relationship with external variables. Future studies with representative samples will be able to determine prevalence figures of food insecurity in different Spanish populations. In general, the relationships of food insecurity scores with all external variables assessed are in line with results from previous studies, providing support for the validity of the questionnaire.

There are some limitations that warrant considerations. Our study included a community sample. As a result, due to insufficient number of cases, we could not analyse the measurement invariance and mean differences for the weight status categories of 'thin' and 'severely thin', while the 'overweight' and 'obese' groups had to be paired together. Another limitation that we must consider is that adolescents self-reported their sociodemographic information. Also, future research could focus on the test-retest reliability of CFSSM-S scores, as our study determined internal consistency only.

However, our study has a number of strengths. The CFSSM-S is the first validated self-reported instrument in Spain, which can be used with psychometric guarantees to measure food insecurity in adolescents. Our study not only assessed the dimensionality of the instrument, but also carried out the measurement invariance, which indicates that the CFSSM-S is a suitable instrument for older children aged 12-17 years of both genders and across a wide range of weight status, SES and FAS categories. Further, the anthropometric measurements of weight and height were not self-reported by adolescents, rather they were collected in situ by trained research staff following a standardized protocol.

\section{Conclusion}

We found the CFSSM-S to be a valid and reliable instrument which can be used by researchers, public health 
professionals and interested non-governmental organizations. Even though the prevalence rate of food insecurity might vary across different regions in Spain and within Catalonia itself, our findings indicate that the Spanish adaptation of CFSSM can be used in different regions of Spain. The CFSSM-S can be employed to assess food insecurity and gain more information about adolescent health in relation to food insecurity in both genders and across a wide age group and different weight status categories, SES and FAS levels in Spanish adolescents.

\section{Acknowledgements}

Acknowledgements: The authors would like to thank the Health and Community Services of the City Council of Terrassa and the Catalan Public Health Agency for facilitating access to the data sample. They are also grateful for the participation and support of the staff and students of the secondary school, IES Torre del Palau. Financial support: This research received no specific grant from any funding agency in the public, commercial or not-for-profit sectors. Conflict of interest: N.S.K., E.P., A.F.D. and D.S.-C. declare no conflicts of interests. Authorship: D.S.-C., A.F.D. and N.S.K. contributed to the study design and data collection procedure. D.S.-C. and A.F.D. carried out the adaptation of the questionnaire into Spanish. E.P. and A.F.D. carried out the statistical analyses. N.S.K. wrote the paper supervised by D.S.-C. except the 'Statistical analysis' and 'Results' sections, which were written by E.P. and A.F.D. The drafts were reviewed by D.S.-C., E.P. and A.F.D. All authors consented to the final manuscript. Ethics of human subject participation: This study was conducted according to the guidelines laid down in the Declaration of Helsinki and all procedures involving human subjects were approved by the ethics committee of Universitat Autònoma de Barcelona. Written informed consent was obtained from both participants and parents of participants.

\section{Supplementary material}

To view supplementary material for this article, please visit https://doi.org/10.1017/S1368980018001672

\section{References}

1. Loopstra R, Reeves A \& Stuckler D (2015) Rising food insecurity in Europe. Lancet 385, 2041.

2. Loopstra R, Reeves A, McKee M et al. (2016) Food insecurity and social protection in Europe: quasi-natural experiment of Europe's great recessions 2004-2012. Prev Med 89, 44-50.

3. Ortiz-Miranda D, Pérez OM \& Alegre EA (2016) Food and nutrition security discursive frames in the context of the Spanish economic crisis. Food Secur 8, 665-677.

4. UNICEF (2017) Building the Future: Children and the Sustainable Development Goals in Rich Countries. Innocenti
Report Card no. 14. https://www.unicef-irc.org/publications/ pdf/RC14_eng.pdf (accessed October 2017).

5. Frazer $\mathrm{H} \&$ Marlier E (2014) Investing in Children: Breaking the Cycle of Disadvantage. A Study of National Policies: Synthesis Report. https://www.liser.lu/publi_viewer.cfm? tmp=2522 (accessed June 2018).

6. Generalitat de Catalunya, Agencia Catalana de Seguridad Alimentaria (2017) Plan de seguridad alimentaria de Cataluña 2017-2021. http://acsa.gencat.cat/web/.content/ 40_Eines_i_recursos/Publicacions/Pla_de_Seguretat_Alimentaria/ 2017-2021/Pla_seguretat_alimentaria_2017_2021.pdf (accessed June 2018).

7. Save the Children España (2017) Desigualtat infantil, igualtat d'oportunitats i polítiques publiques. https://www.savethe children.es/sites/default/files/imce/docs/save_the_childrenanexo_valenciano.pdf (accessed October 2017).

8. Nord M, Andrews M \& Carlson S (2008) Household Food Security in the United States, 2007. Economic Research Report no. ERR-66. https://www.ers.usda.gov/webdocs/ publications/46084/13819_err66fm_1_.pdf?v=4105 (accessed February 2018).

9. Coleman-Jensen A, Rabbitt MP, Gregory CA et al. (2017) Household Food Security in the United States in 2016. Economic Research Report no. ERR-237. https://www.ers. usda.gov/webdocs/publications/84973/err-237.pdf (accessed February 2018).

10. Anderson SA (1990) Core indicators of nutritional status for difficult-to-sample populations. J Nutr 120, 1559-1600.

11. US Department of Agriculture, Economic Research Service (2017) Food Security in the US. Measurement. https://www. ers.usda.gov/topics/food-nutrition-assistance/food-securityin-the-us/measurement/ (accessed April 2018).

12. Barrett CB (2010) Measuring food insecurity. Science 327, 825-828.

13. Kirkpatrick S \& Tarasuk V (2003) The relationship between low income and household food expenditure patterns in Canada. Public Health Nutr 6, 589-597.

14. Tanumihardjo SA, Anderson C, Kaufer-Horwitz $M$ et al. (2007) Poverty, obesity, and malnutrition: an international perspective recognizing the paradox. J Am Diet Assoc 107, 1966-1972.

15. Radimer KL, Olson CM, Greene JC et al. (1992) Understanding hunger and developing indicators to assess it in women and children. J Nutr 24, Suppl. 1, 36S-44S.

16. Coates J, Swindale A \& Bilinsky P (2007) Household Food Insecurity Access Scale (HFIAS) for Measurement of Household Food Access: Indicator Guide (version 3). https://www.fantaproject.org/sites/default/files/resources/ HFIAS_ENG_v3_Aug07.pdf (accessed February 2018).

17. US Department of Agriculture, Economic Research Service (2012) US Household Food Security Survey Module: ThreeStage Design with Screeners. https://www.ers.usda.gov/ media/8271/hh2012.pdf (accessed November 2017).

18. Harrison GG, Stormer A, Herman DR et al. (2003) Development of a Spanish-language version of the US Household Food Security Survey Module. J Nutr 133, 1192-1197.

19. Comité Cientifico de la ELCSA (2012) Escala Latinoamericano y Caribeña de Seguridad Alimentaria (ELCSA): Manual de Uso y Aplicaciones. http://www.fao.org/3/a-i3065s.pdf (accessed February 2018).

20. Fram MS, Frongillo EA, Draper CL et al. (2013) Development and validation of a child report assessment of child food insecurity and comparison to parent report assessment. J Hunger Environ Nutr 8, 128-145.

21. Connell CL, Nord M, Lofton KL et al. (2004) Food security of older children can be assessed using a standardized survey instrument. J Nutr 134, 2566-2577.

22. Hadley C, Lindstrom D, Tessema F et al. (2008) Gender bias in the food insecurity experience of Ethiopian adolescents. Soc Sci Med 66, 427-438. 
23. Shanafelt A, Hearst M, Wang Q et al. (2016) Food insecurity and rural adolescent personal health, home, and academic environments. J Sch Health 86, 472-480.

24. Shtasel-Gottlieb Z, Palakshappa D, Yang F et al. (2015) The relationship between developmental assets and food security in adolescents from a low-income community. I Adolesc Health 56, 215-222.

25. Holben DH \& Taylor CA (2015) Food insecurity and its association with central obesity and other markers of metabolic syndrome among persons aged 12 to 18 years in the United States. J Am Osteopath Assoc 115, 536-543.

26. Casey PH, Simpson PM, Gossett JM et al. (2006) The association of child and household food insecurity with childhood overweight status. Pediatrics 118, e1406-e1413.

27. Dinour LM, Bergen D \& Yeh MC (2007) The food insecurity-obesity paradox: a review of the literature and the role food stamps may play. J Am Diet Assoc 107, 1952-1961.

28. Gundersen C, Garasky S \& Lohman BJ (2009) Food insecurity is not associated with childhood obesity as assessed using multiple measures of obesity. J Nutr 139, 1173-1178.

29. Sarlio-Lahteenkorva S \& Lahelma E (2001) Food insecurity is associated with past and present economic disadvantage and body mass index. J Nutr 131, 2880-2884.

30. Generalitat de Catalunya, Institut d'Estadistica de Catalunya (2017) Estructura i distribució de la població. https:// www.idescat.cat/pub/?id=inddt\&n=916\&lang=es\&by=mun (accessed February 2018).

31. Hollingshead A (1975) Four Factor Index of Social Status, 1st ed. New Haven, CT: Department of Sociology, Yale University.

32. US Department of Agriculture, Economic Research Service (2005) Household Food Security in the United States. https://www.ers.usda.gov/webdocs/publications/45655/29 202_err29g_002.pdf?v=41334 (accessed February 2018).

33. Moreno C, Ramos P, Rivera F et al. (2016) Informe técnico de los resultados obtenidos por el Estudio Health Behaviour in School-aged Children (HBSC) 2014 en España. http:// www.observatoriodelainfancia.es/oia/esp/descargar.aspx?id $=5032 \&$ tipo $=$ documento $($ accessed October 2017).

34. Svedberg P, Nygren JM, Staland-Nyman C et al. (2016) The validity of socioeconomic status measures among adolescents based on self-reported information about parents occupations, FAS and perceived SES; implication for health related quality of life studies. BMC Med Res Methodol 16, 48.

35. World Health Organization (2007) BMI-for-age (5-19 years). http://www.who.int/growthref/who2007_bmi_for_age/en/ (accessed November 2017).

36. International Test Commission (2010) ITC Guidelines for Translating and Adapting Tests. ITC-G-TA-20140617. http://www.psyktestbarn.no/cms/ptb_mm.nsf/lupgraphics/ ITC\%20guidelines.pdf/\$file/ITC\%20guidelines.pdf (accessed June 2018).
37. Muñiz J, Elosua P \& Hambleton RK (2013) International test commission guidelines for test translation and adaptation: second edition. Psicothema 25, 151-157.

38. Jackson DL, Gillaspy JA \& Purc-Stephenson R (2009) Reporting practices in confirmatory factor analysis: an overview and some recommendations. Psychol Methods $\mathbf{1 4}$, 6-23.

39. Brown TA (2006) Confirmatory factor analysis for applied research. In Methodology in the Social Sciences, 1st ed., pp. 214-217 [TD Little, editor]. New York: Guilford Publications.

40. Ezpeleta L \& Penelo E (2015) Measurement invariance of oppositional defiant disorder dimensions in 3-year-old preschoolers. Eur J Psychol Assess 31, 45-53.

41. McDonald RP (1999) Test Theory: A Unified Treatment. Hillsdale, NJ: Erlbaum.

42. Abad FJ, Olea J, Ponsoda V et al. (2011) Medición en Ciencias Sociales y de la Salud. Madrid: Síntesis.

43. Tarasuk V (2001) Discussion Paper on Household Food Insecurity. http://www.hc-sc.gc.ca/fn-an/alt_formats/hpfbdgpsa/pdf/nutrition/food_sec_entire-sec_aliments_entier-eng. pdf (accessed February 2018).

44. Nord M (2009) Food Insecurity in Households with Children: Prevalence, Severity, and Household Characteristics. Economic Information Bulletin no. EIB-56. https://www.ers. usda.gov/webdocs/publications/44419/9360_eib56_1_.pdf? $\mathrm{v}=41055$ (accessed February 2018).

45. Eisenmann JC, Gundersen C, Lohman BJ et al. (2011) Is food insecurity related to overweight and obesity in children and adolescents? A summary of studies, 1995-2009. Obes Rev 12, e73-e83.

46. Nguyen BT, Ford CN, Yaroch AL et al. (2017) Food security and weight status in children: interactions with food assistance programs. Am J Prev Med 52, 2S2, S138-S144.

47. Tester J, Lang T \& Laraia BA (2016) Disordered eating behaviours and food insecurity: a qualitative study about children with obesity in low-income households. Obes Res Clin Pract 10, 544-552.

48. Olson CM, Bove CF \& Miller EO (2007) Growing up poor: long-term implications for eating patterns and body weight. Appetite 49, 198-207.

49. Wight V, Kaushal N, Waldfogel J et al. (2014) Understanding the link between poverty and food insecurity among children: does the definition of poverty matter? J Child Poverty 20, $1-20$.

50. Loopstra R \& Tarasuk V (2013) What does increasing severity of food insecurity indicate for food insecure families? Relationships between severity of food insecurity and indicators of material hardship and constrained food purchasing. J Hunger Environ Nutr 8, 337-349.

51. Muthén LK \& Muthén BO (1998-2017) Mplus User's Guide, 8th ed. Los Angeles, CA: Muthén \& Muthén. 